\title{
Peripheral Dentinogenic Ghost Cell Tumor: A Rare Odontogenic Entity Hidden within the Ordinary Clinical Features of Reactive Gingival Lesions
}

\author{
Souliou $\mathrm{X}^{1, *}$, E. Chrysomali ${ }^{2}$, X. Papadhmas ${ }^{3}$, F. Tzerbos ${ }^{4}$ \\ ${ }^{1}$ School of Dentistry, National and Kapodistrian University of Athens, Greece \\ ${ }^{2}$ Assistant Professor, Department of Oral Medicine and Pathology, School of Dentistry, National and Kapodistrian University of \\ Athens, Greece \\ ${ }^{3}$ Department of Oral and Maxillofacial Surgery, School of Dentistry, National and Kapodistrian University of Athens, Greece \\ ${ }^{4}$ Assistant Professor, Department of Oral and Maxillofacial Surgery, School of Dentistry, National and Kapodistrian University of \\ Athens, Greece \\ *Corresponding author: xrensouliou@hotmail.com
}

Received February 11, 2015; Revised March 20, 2015; Accepted April 02, 2015

\begin{abstract}
The extraosseous odontogenic tumors may raise diagnostic challenges due to their imitation for reactive gingival lesions. Among them, the peripheral dentinogenic ghost cell tumor (PDGCT) is an extremely rare benign odontogenic neoplasm with up to 30 cases published in the literature. PDGCT occurs in older adults most commonly in the anterior mandibular region. We present a new case of PDGCT located in the maxillary premolar gingiva of a 67- year old man along with differential diagnosis. The lesion was presented as a broad based tumor covered by ulcerated mucosa. The histopathologic examination revealed the typical features of PDGCT exhibiting multiple irregular masses of ghost cell aggregates, dysplastic dentin in close relation to ameloblastoma-like epithelial islands. Despite of the rarity of this entity, PDGCT should be included in the differential diagnosis of the gingival tumors.
\end{abstract}

Keywords: dentinogenic ghost cell tumor, peripheral, odontogenic tumors, benign odontogenic neoplasm, maxilla, case report

Cite This Article: Souliou X, E. Chrysomali, X. Papadhmas, and F. Tzerbos, "Peripheral Dentinogenic Ghost Cell Tumor: A Rare Odontogenic Entity Hidden within the Ordinary Clinical Features of Reactive Gingival Lesions.” International Journal of Dental Sciences and Research, vol. 3, no. 3 (2015): 48-51. doi: 10.12691/ijdsr3-3-2.

\section{Introduction}

Odontogenic neoplasms with ghost cells are rare lesions which under the WHO classification terminology include different in biologic behaviour entities: the calcifying cystic odontogenic tumor (CCOT), the dentinogenic ghost cell tumor (DGCT) and the ghost cell odontogenic carcinoma (GCOC). [1,2] CCOT is a benign cystic neoplasm characterized by an ameloblastoma-like epithelium with ghost cells that may calcify. DGCT is a locally invasive odontogenic neoplasm characterised by ameloblastoma-like islands of epithelial cells in a mature connective tissue stroma, while aberrant keratinization may be found in the form of ghost cells in association with varying amounts of dysplastic dentin. GCOC is a malignant neoplasm that may be developed due to malignant transformation of a pre-existing benign CCOT $[1,2,3]$.

DGCT comprising the $1.9 \%$ to $2.1 \%$ of all odontogenic tumors may arise either as an extra-osseous (peripheral) or as intra-osseous (central) lesion [1,2]. Although both DGCT variants share similar histopathological features, the central variant seems to be more aggressive locally with frequent recurrences (28\%) compared to the peripheral one that appears no recurrence [4]. The peripheral DGCT (PDGCT) is extremely rare odontogenic neoplasm, and in a recent review of the English literature the number of the published PDGCT cases did not exceed the thirty [4]. The mandible is more commonly involved than maxilla with the anterior region reported as a frequent location of this lesion [1,2,3,4]. Radiographic findings limited to a superficial erosion (saucerization) of the underlying bone may be seen [4,5]. The clinical features of the PDGCT are not indicative mimicking reactive in nature, inflammatory lesions or other odontogenic tumors that can arise in the gingival mucosa.

The purpose of this article is to present a new case of peripheral dentinogenic ghost cell tumor analyzing the differential diagnosis.

\section{Case Report}

A 67 year old male was referred to a private Oral and Maxillofacial clinic for evaluation of an asymptomatic gingival swelling which was in the area of left upper 
premolars. The patient was not a smoker and there were no known allergies, his medical history included noninsulin depended diabetes, hypertension and thyroid disease; all of the above were controlled with systematic medication. The patient noticed the swelling approximately one month before his visit. According to him, since that time a slight growth of the size of the lesion occurred. Upon clinical examination, a $2 \mathrm{~cm}$ in its wide diameter well circumscribed, red in color, firm in consistency and painless in palpation mass was detected in the area of the left maxillary buccal attached gingiva. The lesion was ulcerative and a fibrin clot over the ulcer was evident, while the adjacent teeth $(\# 24,25)$ were vital (Figure 1). No signs of lymphadenopathy were found.

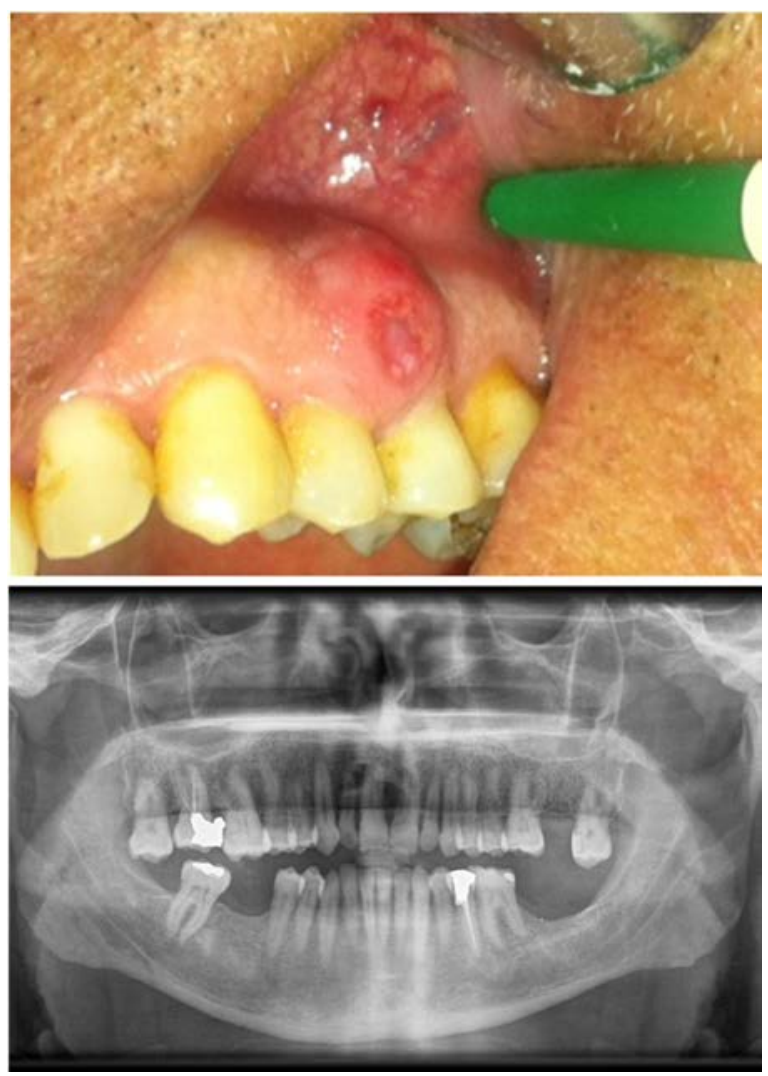

Figure 1. Clinical appearance of the lesion at the time of first examination with no evidence of radiologic findings in the left maxillary premolar region

The panoramic radiograph revealed no signs of pathology (Figure 2) and a surgical intervention for total biopsy of the lesion was decided. Under local anesthesia, a full- thickness mucoperiostel flap was elevated and total excision of the lesion was performed till "clear" clinical borders, A careful curettage of the underlying bone followed with special attention to the adjacent teeth. The surgical area was irrigated and the exposed alveolar bone was covered with a sliding mucoperiostel flap. The flap was sutured using 3,0 vicryl sutures. The specimen was submitted to histological examination. The patient was prescribed antibiotic (amoxicillin $500 \mathrm{mg} / \mathrm{per}$ os) an analgesic (paracetamol $500 \mathrm{mg} / \mathrm{per}$ os) medication. The postoperative course was uneventful and six months after the operation there were no signs of recurrence.

The surgical specimen (Figure 2a) was fixed in 10\% neutral formalin and processed for histopathological analysis. Gross examination of the submitted specimen showed a whitish-grey tissue firm in consistency measuring approximately 2.5X2X1 cm. The histopathologic analysis revealed a non capsulated solid tumor comprising multiple irregular masses of ghost cell aggregates, dysplastic dentin or osteodentin like material in close relation to epithelial cords and islands of ameloblastic-like proliferations. The epithelial islands exhibited a peripheral row of cuboidal or columnar cells with polarized nuclei enclosing more loosely arranged spindle shaped cells, while some of them showed focal microcystic changes. The ghost cells displayed pale cytoplasm with no nuclei or faint nuclear outline (Figure 2). Multinucleated giant cells associated with ghost cells and few foci of basophilic dystrophic calcifications were evident in the stroma, whereas scattered inflammatory cells infiltrates and hemosiderin deposits were additionally seen. The overlying keratinized epithelium was focally thin, but a direct continuation of the basal cells with the tumor epithelial islands was not evident (Figure 2). Based on the histopathological features, diagnosis of a peripheral dentinogenic ghost cell tumor was rendered.

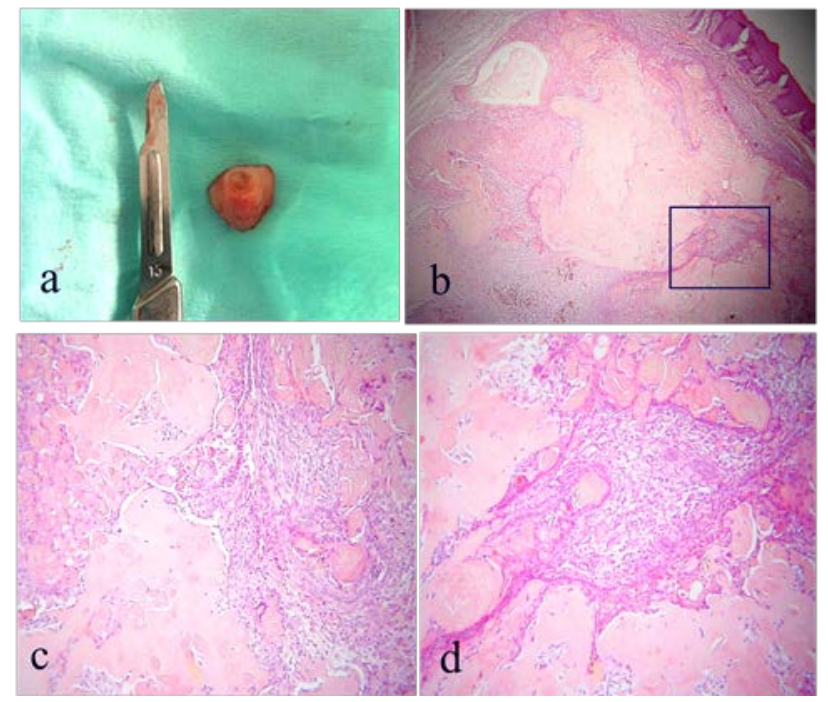

Figure 2. (a) surgical specimen of the lesion. (b) The solid morphology of the tumor exhibiting irregular masses of dysplastic dentin like material with few focal cystic changes. (c) Ghost cell aggregates admixed with dysplastic dentin/osteodentin (d) higher power view (of above square) showing dysplastic dentin/osteodentin surrounded by ameloblastic-like epithelium within a loose fibrous stroma with scattered inflammatory cells. HXE (b) X250, (c) and (d) X400

\section{Discussion}

In the present case, the clinical description indicated a slow growing, asymptomatic lesion located on the buccal gingiva of the premolar maxillary region that showed ulcerated surface. A wide range of differential diagnoses including mainly the commonly seen reactive in nature lesions, such as peripheral giant cell granuloma, pyogenic granuloma, peripheral ossifying fibroma or peripheral odontogenic tumors, such as ameloblastoma, keratocystic odontogenic tumor, CCOT, odontogenic fibroma, ameloblastic fibroma, adenomatoid odontogenic tumor which may present as gingival broad based masses can be considered. The location of the above mentioned lesions are very likely to develop in the maxillary gingival mucosa. However, peripheral ameloblastoma may usually 
arise in the posterior mandibular region [6] and giant cell granuloma may show a slight predilection for the mandible as well [7]. In addition, both of these lesions occur often during $5^{\text {th }}$ or $6^{\text {th }}$ decade of life, whereas peripheral ossifying fibroma and pyogenic granuloma may be seen at any age, but most commonly present in adolescents or young adults [7].

The CCOT and the keratocystic odontogenic tumor may also occur in gingiva at any age, but keratocystic odontogenic tumor usually present in older adults, whereas other rare peripheral odontogenic tumors including, ameloblastic fibroma and adenomatoid odontogenic tumor may represent hamartomatous conditions of infants and children [8]. In our patient, based on the absence of radiological findings, the clinical features of the lesion and the patient age the provisional diagnosis was maxillary peripheral giant cell granuloma. Clinically, the PDGCT presenting as epulis-like or exophitic enlargement with ulcerated red surface exhibits similarity with reactive or odontogenic lesions causing difficulty in its clinical diagnosis. Thus, PDGCT is usually revealed only after the excisional biopsy and histopathologic examination of the lesion.

The ghost cell odontogenic neoplasms are rare jaw tumors. In a recent study in an Iranian population, the researchers by searching 14,106 patient files during a period of 44 years found only 37 cases of ghost cell odontogenic tumors; PDGCT accounted for only 10,81\% of them [5]. Pommer et al 2013 [4] in their meta-analytic literature update disclosed 28 well-documented cases and showed that males are affected more commonly than females, while the patient mean age is estimated 59 years old. The absence of bone involvement seems to be usual, but slight bone erosion can be found on radiographic examination in more than half of the published cases (53\%) [4]. Clinically, the PDGCT presents as a swelling with the canine till first molar area to be more frequent and a certain predilection for the mandible. The maxillary location of the PDGCT is referred in a percentage of $26 \%$ [9]. The majority of patients report no pain or mild discomfort as a symptom [4,5].

PDGCT is derived from dental lamina remnants in the gingiva $[1,2,3]$. The exact etiologic factors involved in the development of this tumor remain elusive. Trauma or/and chronic irritation may be considered as predisposing factor, since 7 cases of PDGCT have been described in edentulous alveolar ridge in denture wearers [9,10,11], whereas a PDGCT case has been reported in the maxillary gingiva at tooth extraction site [12]. In our patient, a local predisposing factor was not evident. However, it was unknown if the cover mucosa ulceration was related to a secondary trauma or not; thus the role of chronic irritation cannot be totally excluded. The connection between the basal cells of the overlying oral mucosa epithelium and the tumor epithelial islands has been suggested that may be involved in the histogenesis of the PDGCT [9]. This finding was not observed in our case. According to Buchner et al [13], if PDGCT cases show no evidence of such a connection, then might be derived from the rest of Serres.

PDGCT demonstrates histologically ameloblastomalike epithelial islands in a fibrous connective tissue stroma. A misdiagnosis could be made specifically due to its similarity with the ameloblastic tissue islands seen in peripheral ameloblastoma. The distinctive features that distinguish PDGCT from other odontogenic tumors, such as peripheral ameloblastoma and peripheral calcifying epithelial odontogenic tumor are the presence of ghost cells, dysplastic dentin, and dystrophic calcifications $[1,2,3,14]$. The ghost cells of PDGCT exhibiting positivity for pan-cytokeratin and cytokeratin-14 may represent transformed epithelial cells of unknown origin characterized by abnormal keratinization [14,15].

In contrast to the central DGCT, which may display high rate of recurrences even a malignant transformation suggesting a surgical management similar to ameloblastoma [16], the PDGCT demonstrates a very good biological behavior. Although no recurrences or malignant potential have been reported, patient should remain in a close follow-up. Conservative curettage and enucleation seem to be appropriate as a treatment of choice for PDGCT [1-5].

In conclusion, a case of peripheral DGCT was presented that showed the typical histopathologic features of this benign odontogenic neoplasm. Despite of the rarity of this entity, PDGCT should be included in the differential diagnosis of the gingival tumors.

\section{References}

[1] Barnes L, Eveson JW, Reichart P, Sidransky D, editors. World Health Organization classification of tumours. Pathology and genetics of head and neck tumours. Lyon: IARC Press; 2005. p. 284.

[2] Ledesma-Montes C, Gorlin RJ, Shear M, Praetorius F, MosquedaTaylor A, Altini M, Unni K, Paes de Almeida O, Carlos-Bregni R, Romero de León E, Phillips V, Delgado-Azañero W, MenesesGarcía A. International collaborative study on ghost cell odontogenic tumours: calcifying cystic odontogenic tumour, dentinogenic ghost cell tumour and ghost cell odontogenic carcinoma. J Oral Pathol Med 2008; 37: 302-8.

[3] Colmenero C, Patron M, Colmenero B. Odontogenic ghost cell tumours. The neoplastic form of calcifying odontogenic cyst. J Craniomaxillofac Surg 1990; 18: 215-8.

[4] Pommer B, Dvorak G, Rappersberger K, Jordan RCK, Watzek G, Pogrel MA. Pyogenic granuloma mimicking peripheral dentinogenic ghost-cell tumour recurrence: case report and metaanalytic literature update. Oral Surgery 6: 98-103, 2013.

[5] Etemad-Moghadam S, Baghaee F, Dadafarid Z, Alaeddini M. A 44-year analysis of ghost cell odontogenic tumour subtypes in an Iranian population. J Cranio Maxillofac Surg 42: 1154-9, 2014.

[6] Philipsen HP, Reichart PA. Nikai H, Takata T, Kudo Y. Peripheral ameloblastoma: biological profile based on 160 cases from the literature Oral Oncology 2001 37: 17-27.

[7] Neville BW, Damm DD, Allen CM, Bouquot JE. Oral and Maxillofacial Pathology. St Louis, MO: Saunders Elsevier; 2009.

[8] Ide F, Mishima K, Saito I, Kusama K. Rare peripheral odontogenic tumors: report of 5 cases and comprehensive review of the literature. Oral Surg Oral Med Oral Pathol Oral Radiol Endod 2008; 106: e22-e28.

[9] Bello I, Qannam A, Al-Zahrani A, AlDosari A. Peripheral Dentinogenic Ghost Cell Tumor: Report of a Case and Literature Review Int J Surg Pathol 20: 494-9, 2012.

[10] Hong SP, Ellis GL, Hartman KS. Calcifying odontogenic cyst. A review of ninety two cases with reevaluation of their nature as cyst or neoplasm, the nature of the ghost cells, and subclassification. Oral Surg Oral Med Oral Pathol. 1991; 72: 56-64.

[11] Kamat SS, Diwakar GS, Ahmed MB, Shetty PR. Peripheral dentinogenic ghost cell tumor. Med J DY Patil Univ 2013; 6: 4369.

[12] Wong YK, Chiu SC, Pang SW, Cheng JCF. Peripheral dentinogenic ghost cell tumour presenting as a gingival mass. $\mathrm{Br} \mathrm{J}$ Oral Maxillofac Surg 2004 42: 173-5. 
[13] Buchner A, Merell PW, Hansen LS, Leider AS. Peripheral (extraosseous) calcifying odontogenic cyst. A review of forty five cases. Oral Surg Oral Med Oral Pathol 1991; 72: 65-70.

[14] Lee SK, Kim YS, Current Concepts and Occurrence of Epithelial Odontogenic Tumors: II. Calcifying Epithelial Odontogenic Tumor Versus Ghost Cell Odontogenic Tumors Derived from Calcifying Odontogenic Cyst. The Korean J Pathol 2014; 48: 175187.
[15] Candido GA, Viana KA, Watanabe S, Vencio EF. Peripheral dentinogenic ghost cell tumor: a case report and review of the literature. Oral Surg Oral Med Oral Pathol Oral Radiol Endod 2009; 108:-e90.

[16] Sun G, Huang X, Hu Q, Yang X, Tang E. The diagnosis and treatment of dentinogenic ghost cell tumor. Int J Oral Maxillofac Surgery 2009 38: 1179-83. 